\title{
Transarterial chemoembolization with drug- eluting beads versus conventional transarterial chemoembolization in locally advanced hepatocellular carcinoma
}

\author{
This article was published in the following Dove Press journal: \\ Hepatic Medicine: Evidence and Research \\ 20 June 2016 \\ Number of times this article has been viewed
}

\author{
Johannes Baur' \\ Christian O Ritter ${ }^{2}$ \\ Christoph-Thomas \\ Germer' \\ Ingo Klein' \\ Ralph Kickuth ${ }^{2}$ \\ Ulrich Steger ${ }^{\prime}$ \\ 'Department of General, Visceral, \\ Vascular, and Pediatric Surgery, \\ ${ }^{2}$ Institute of Radiology, University \\ Hospital Würzburg, Würzburg, \\ Germany
}

\begin{abstract}
Purpose: In hepatocellular carcinoma patients with large or multinodal tumors, where curative treatment options are not feasible, transarterial therapies play a major role. Transarterial chemoembolization (TACE) with drug-eluting beads (DEB-TACE) is a promising new approach due to higher intratumoral and lower systemic concentration of the chemotherapeutic agent compared to conventional TACE (cTACE).

Patients and methods: In a retrospective analysis, 32 patients with hepatocellular carcinoma who received either DEB or a cTACE were compared regarding survival time, disease recurrence, and side effects such as pain and fever.

Results: No significant differences could be detected between the cTACE and DEB-TACE groups with regard to mean hospital stay, appearance of postinterventional fever, or 30-day mortality. However, the application of intravenous analgesics as postinterventional pain medication was needed more often in patients treated with DEB-TACE ( $57.1 \%$ vs $12.5 \%, P=0.0281)$. The overall median survival after the initial procedure was 10.8 months in the cTACE group and 9.2 months in the DEB-TACE group, showing no significant difference.

Conclusion: No survival benefit for patients treated with either DEB-TACE or cTACE was observed. Surprisingly, a higher rate of postinterventional pain could be detected after DEBTACE.

Keywords: transarterial chemoembolization, hepatocellular carcinoma, drug-eluting beads
\end{abstract}

\section{Introduction}

Only $10 \%-20 \%$ of patients with hepatocellular carcinoma (HCC) have the option for curative therapy such as resection, transplantation, or local ablation. ${ }^{1}$ According to the Barcelona clinic liver cancer tumor staging, transarterial chemoembolization (TACE) is performed in patients with large or multinodal tumors where curative treatments are not feasible. However, these patients need to have a sufficient remaining liver function (Child-Pugh class A and B liver cirrhosis) and absence of extrahepatic tumor manifestation, macrovascular invasion, and cancer-related symptoms (Barcelona clinic liver cancer intermediate stage B). ${ }^{2,3}$ There is evidence that TACE is able to improve survival in these patients compared to symptomatic treatments alone. ${ }^{4-6}$ Like every invasive treatment, TACE bears the risk of side effects. The most common adverse events are abdominal pain, nausea, fever, and elevated transaminases during the initial days post intervention. Severe side effects such as hepatic decompensation, gastrointestinal bleeding, or treatment-related death are found in $<10 \%$ of the patients treated with TACE. ${ }^{2,3}$
Correspondence: Johannes Baur Department of General,Visceral,Vascular and Pediatric Surgery, University Hospital Würzburg, Oberdürrbacherstraße, 97080 Würzburg, Germany

Tel +49 931 2013 8812

Email baur_j@ukw.de 
During TACE, a chemotherapeutic agent is administered into the feeding artery of the HCC nodule followed by application of an embolic material to gain a high intratumoral concentration of chemotherapeutics and tumor necrosis. ${ }^{\text {? }}$ Nonresorbable drug-eluting beads (DEB) are new embolic agents used in TACE. First studies showed higher intratumoral and lower systemic concentration of doxorubicin compared to the common doxorubicin-lipiodol mixture, which is used as an embolic agent in conventional TACE (cTACE). ${ }^{8-10}$ Prospective randomized controlled trials comparing cTACE with DEB-TACE showed less systemic side effects of the chemotherapeutic agent but no superiority in tumor response or overall survival after DEB-TACE., ${ }^{71}$ However, some retrospective studies showed better treatment response and longer time to progression after DEB-TACE. ${ }^{12,13}$

Our retrospective study was conducted to compare tumor response and survival and postinterventional side effects such as pain and fever that influence the postinterventional quality of life in HCC patients treated with either cTACE or DEB-TACE.

\section{Patients and methods}

\section{Patients}

Data of 32 patients suffering from unresectable HCC and treated with TACE were retrospectively analyzed. HCC was diagnosed according to the American Association for the Study of Liver Diseases Practice Guidelines with one or two dynamic imaging techniques. In case of doubt, a biopsy was performed. ${ }^{3}$ The protocol for this study was approved by the ethics committee of the University of Wuerzburg; as such, no written informed consent from each individual patient was required for inclusion to this study, since it was retrospective, and all data was de-identified. Procedural consent for TACE was obtained from each patient.

Patient's inclusion criteria were 1) diagnosed HCC outside the Milan criteria (one single lesion $<5 \mathrm{~cm}$ or three lesions $<3 \mathrm{~cm} \mathrm{each}^{14}$ ) and 2) treatment with DEB-TACE or cTACE. Patients were excluded from the analysis if 1 ) the tumor was inside the Milan criteria; 2) the patients were consecutively treated with curative procedures (transplantation, resection, or local ablative methods) after initial TACE; or 3) the patients received selective internal radiation therapy after initial TACE. As the treatment with targeted therapy like sorafenib is a common part in the treatment of advanced HCC nowadays, patients receiving this form of medication were not excluded from the study. No patient received sorafenib before or during the first TACE procedure. Sorafenib was administered only in case of recurrent disease not feasible for TACE or as additive therapy after TACE. Decision for targeted therapies was made by interdisciplinary tumor board consensus.

\section{Procedure}

As described elsewhere, each procedure was performed via femoral artery puncture. ${ }^{12,15}$ First, standardized pressure arteriography of celiac and superior mesenteric arteries was performed to investigate arterial anatomy of the liver, vascular supply of tumor nodes, and patency of the portal vein. Chemotherapeutics and embolic agents were infused either superselectively into the tumor-supplying arteries or nonselectively into a single liver lobe or the whole organ according to tumor size and location. A superselective procedure was performed in $33.3 \%$ of the patients in the cTACE group and $21.4 \%$ of patients in the DEB-TACE group.

For cTACE, a mixture of doxorubicin $(\mathrm{n}=11)$ or epirubicin $(n=7)$ and Lipiodol was used. For DEB-TACE, doxorubicin was applied in nine cases and epirubicin in five. Dosages depended on body surface area and tumor size. The choice of chemotherapeutic agent and the decision of cTACE versus DEB-TACE were at the discretion of the performing radiologist.

For DEB-TACE, DEB (DC Bead; Biocompatibles, Farnham, UK) with a diameter of 300-500 $\mu$ m were used. According to the body surface area and tumor size, the concentration of the used chemotherapeutic drug was determined. Embolization with microspheres (Bead Block; Biocompatibles) after cTACE or DEB-TACE application was a facultative treatment to consolidate stasis within the feeding arteries.

\section{Study outcomes}

The primary outcomes of the study were 30-day mortality, peri-interventional need of pain medication, fever $\geq 38.0^{\circ} \mathrm{C}$ $\left(100.4^{\circ} \mathrm{F}\right)$, and elevation of transaminases to evaluate the appearance of a postembolization syndrome after cTACE or DEB-TACE. Another primary outcome of the study was overall and median survival after the initial TACE.

The secondary outcomes were local and extrafocal recurrence of the disease after 3 months and 6 months detected in radiologic imaging, which is defined as newly detected areas of contrast enhancement at the edge of the known HCC nodules or newly detected intra- or extrahepatic manifestation of HCC.

The patients were usually followed up every 3 months after the initial TACE with the same cross-sectional multiphase imaging used before the initial TACE (multislice computed tomography or dedicated magnetic resonance imaging). The follow-up cases and their further treatment were discussed in an interdisciplinary conference.

\section{Statistical analysis}

A $P$-value $<0.05$ was defined to be significant. A unpaired $t$-test was performed to investigate continuous variables, and 
a chi-square test was used to investigate categorical variables. The Kaplan-Meier method was used to estimate the overall and median survival.

\section{Results}

Thirty-two patients treated with TACE met the abovementioned inclusion criteria. Patients' characteristics are shown in Table 1 . In all, 18 patients $(56.3 \%)$ received cTACE and 14 patients (43.8\%) DEB-TACE. There were no differences due to sex, age, tumor burden, Child-Pugh stage, Okuda stage, and initial Model for End-stage Liver Disease score. In average, 1.61 procedures per patient in the cTACE group and 1.71 procedures per patient in the DEB-TACE group have been performed. A total of ten patients $(55.6 \%)$ in the cTACE group and two patients $(14.3 \%)$ in the DEB-TACE group received additional bland embolization. None of the patients received sorafenib before or during the first TACE procedure. In all, $33.3 \%$ of patients in the cTACE group and $57.1 \%$ of patients in the DEB-TACE group received sorafenib in the course after the first TACE $(P=0.1780)$.

\section{Peri-interventional adverse events}

There was no statistically significant difference between cTACE and DEB-TACE with regard to 30-day mortality. In all, three patients died within 30 days after initial TACE, two in the cTACE group (11.1\%) and one in DEB-TACE group $(7.1 \%)$. The reasons for death were gallbladder rupture with consecutive abdominal sepsis and decompensation of liver cirrhosis in the cTACE group and rupture of the tumor with consecutive hemorrhagic shock in the DEB-TACE group. Between the cTACE and DEB-TACE groups, no statistically significant differences could be detected with regard to mean hospital stay (3.7 days vs 3.0 days) and appearance of postinterventional fever of $\geq 38.0^{\circ} \mathrm{C}\left(100.4^{\circ} \mathrm{F}\right)$ within the first 3 days after intervention (50.0\% vs 50.0\%). In all, five patients $(31.3 \%)$ in the cTACE group and ten patients $(71.1 \%)$ in the DEB-TACE group required postinterventional application of analgesics $(P=0.0281)$. Intravenous analgesics as well as opiates were also needed more often in patients treated with DEB-TACE (Table 2). Additionally, DEB-TACE patients did show a trend toward a higher increase in transaminase levels within 3 days after intervention, although differences did not reach statistical significance (data not shown).

\section{Median and overall survival}

Figure 1 shows the overall survival after initial TACE of patients included in this study. A difference or a trend toward superiority of one of the procedures in the overall survival after initial TACE could not be detected. The overall median survival after the initial procedure was 10.8 months in the

Table I Characteristics of study collective

\begin{tabular}{|c|c|c|c|c|}
\hline Characteristics & Overall & cTACE & DEB-TACE & $P$-value \\
\hline \multicolumn{5}{|l|}{ Patients, n (\%) } \\
\hline Total & $32(100)$ & $18(56.3)$ & $14(43.8)$ & 0.854 \\
\hline Male & $30(93.8)$ & $17(94.4)$ & $13(92.9)$ & \\
\hline Female & $2(6.3)$ & I (5.6) & I (7.I) & \\
\hline \multicolumn{5}{|l|}{ Age (years) } \\
\hline Mean & 66.8 & 68.5 & 64.7 & 0.2613 \\
\hline \multicolumn{5}{|l|}{ Tumor burden, n (\%) } \\
\hline Unilobar & $12(37.5)$ & $7(38.8)$ & $5(35.7)$ & 0.854 \\
\hline Bilobar & $20(62.5)$ & II (6I.I) & $9(64.3)$ & \\
\hline Multinodular ( $\geq 4$ nodes) & $19(59.4)$ & $8(44.4)$ & II (78.6) & 0.0512 \\
\hline \multicolumn{5}{|l|}{ Okuda stage, ${ }^{\mathrm{a}}$ n (\%) } \\
\hline 1 & $12(40.0)$ & $7(43.8)$ & $5(35.7)$ & 0.7398 \\
\hline II & $15(50.0)$ & $7(43.8)$ & $8(57.1)$ & \\
\hline III & $3(10.0)$ & $2(12.5)$ & I (7.I) & \\
\hline \multicolumn{5}{|l|}{ Child-Pugh class, ${ }^{a}$ n (\%) } \\
\hline$A$ & $21(67.7)$ & $10(58.8)$ & II (78.6) & 0.3200 \\
\hline B & $8(25.8)$ & $5(29.7)$ & $3(21.4)$ & \\
\hline $\mathrm{C}$ & $2(6.5)$ & $2(11.8)$ & $0(0.0)$ & \\
\hline \multicolumn{5}{|l|}{ MELD score ${ }^{a}$} \\
\hline Mean & 10.0 & II.I & 8.8 & 0.0771 \\
\hline \multicolumn{5}{|l|}{ Targeted therapy, n (\%) } \\
\hline Sorafenib after initial TACE & $14(4 \mid .4)$ & $6(33.3)$ & $8(57.1)$ & 0.1780 \\
\hline
\end{tabular}

Note: ${ }^{a}$ In up to two cases, patients could not be classified due to missing data.

Abbreviations: CTACE, conventional transarterial chemoembolization; DEB-TACE, transarterial chemoembolization with drug-eluting beads; MELD, Model for End-stage Liver Disease; TACE, transarterial chemoembolization. 
Table 2 Morbidity and mortality after cTACE and DEB-TACE

\begin{tabular}{|c|c|c|c|c|}
\hline Postinterventional course & Overall & cTACE & DEB-TACE & $P$-value \\
\hline \multicolumn{5}{|l|}{ Mortality } \\
\hline Within 30 days, $n(\%)$ & $3(9.4)$ & $2(11.1)$ & I (7.I) & 0.7024 \\
\hline \multicolumn{5}{|l|}{ Analgetic requirements } \\
\hline Data available, $n$ & 30 & 16 & 14 & \\
\hline Pain medication applied, n (\%) & $15(50.0)$ & $5(3 \mid .3)$ & $10(7 \mathrm{I} .1)$ & $0.028 I$ \\
\hline iv application, n (\%) & $10(33.3)$ & $2(12.5)$ & $8(57.1)$ & 0.0097 \\
\hline Opiate, n (\%) & $10(33.3)$ & $2(12.5)$ & $8(57.1)$ & 0.0097 \\
\hline \multicolumn{5}{|l|}{ Pyrexia } \\
\hline Data available, $\mathrm{n}$ & 27 & 16 & 14 & \\
\hline$\geq 38.0^{\circ} \mathrm{C} / 100.4^{\circ} \mathrm{F}, \mathrm{n}(\%)$ & $15(55.6)$ & $8(50.0)$ & $7(50.0)$ & 1.0000 \\
\hline \multicolumn{5}{|l|}{ Hospital stay after TACE (d) } \\
\hline Mean & 3.2 & 3.7 & 3.0 & 0.2122 \\
\hline
\end{tabular}

Abbreviations: cTACE, conventional transarterial chemoembolization; DEB-TACE, transarterial chemoembolization with drug-eluting beads; iv, intravenous; TACE, transarterial chemoembolization; d, days.

cTACE group and 9.2 months in the DEB-TACE group, showing no statistically significant difference.

Therefore, a substratification in the following subgroups has been performed: bilobar tumor burden, multinodular tumor burden ( $\geq 4$ tumor nodules), Child-Pugh class A cirrhosis, and not superselective procedure (TACE of an entire liver lobe or whole liver). In none of the subgroups, superiority of a single procedure in the overall or median survival after the initial TACE could be detected (Table 3). Yet, in case of multinodular tumor burden in patients with

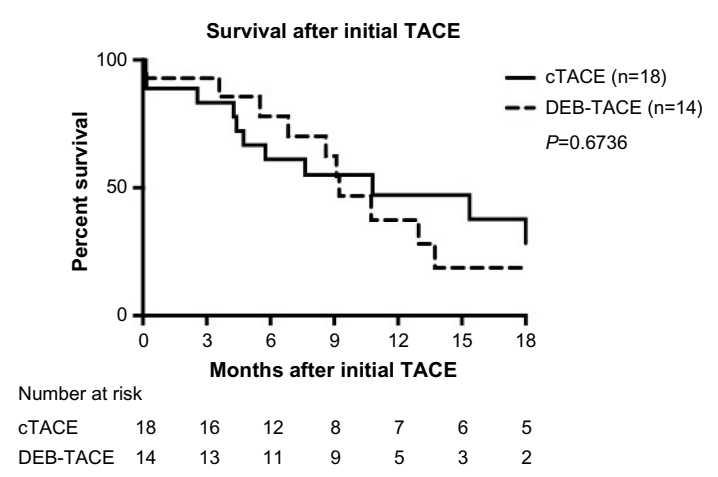

Figure I Overall survival after initial cTACE and DEB-TACE.

Abbreviations: TACE, transarterial chemoembolization; cTACE, conventional transarterial chemoembolization; DEB-TACE, transarterial chemoembolization with drug-eluting beads.
Child-Pugh class A cirrhosis, a trend toward longer median survival in cTACE could be detected.

\section{Radiological recurrence of disease}

Local or extrafocal recurrence of HCC 3 months and 6 months after the initial TACE as defined earlier is shown in Table 4. No differences between the cTACE and DEBTACE could be detected. However, there was a trend toward a higher rate of extrafocal recurrence in patients treated with DEB-TACE.

\section{Discussion}

For patients with $\mathrm{HCC}$ not suitable for curative treatment options, transarterial therapy with chemotherapeutic DEB is a new alternative for locoregional treatment. Several preclinical and clinical studies showed high intratumoral and low systemic dosages of doxorubicin ${ }^{8,10,16}$ resulting in a satisfying safety profile and high rates of tumor response. ${ }^{17,18}$ Although these studies showed promising results, prospective randomized controlled trials could not prove significant superiority of DEB-TACE over cTACE regarding tumor response in the overall setting. ${ }^{7,11}$ However, less drug-related side effects, eg, fever, liver toxicity events, cardiotoxicity, and alopecia, after the application of DEB were observed. ${ }^{7,15}$

Table 3 Median survival after cTACE and DEB-TACE

\begin{tabular}{llllr}
\hline $\begin{array}{l}\text { Median survival } \\
\text { (months after initial TACE) }\end{array}$ & cTACE & DEB-TACE & Ratio & $\mathbf{9 5 \% ~ C I ~ o f ~ r a t i o ~}$ \\
\hline Overall & 10.8 & 9.2 & 1.170 & $(0.5054-2.707)$ \\
Multinodular tumor burden & 15.1 & 10.7 & 1.410 & $(0.4612-4.310)$ \\
Bilobar tumor burden & 15.4 & 13.0 & 1.158 & $(0.3822-3.675)$ \\
Child-Pugh class A & 22.1 & 10.7 & 2.061 & $(0.6540-6.492)$ \\
Not superselective procedure & 10.8 & 10.7 & 1.007 & $(0.3778-2.682)$ \\
\hline
\end{tabular}

Abbreviations: cTACE, conventional transarterial chemoembolization; DEB-TACE, transarterial chemoembolization with drug-eluting beads; TACE, transarterial chemoembolization; $\mathrm{Cl}$, confidence interval. 
Table 4 Recurrence of disease after cTACE and DEB-TACE

\begin{tabular}{|c|c|c|c|c|}
\hline & Overall & cTACE & DEB-TACE & $P$-value \\
\hline \multicolumn{5}{|l|}{3 months after initial TACE } \\
\hline Number of patients in follow-up & 26 & 15 & 11 & \\
\hline Local recurrence, $\mathrm{n}(\%)$ & $16(61.5)$ & $9(60.0)$ & $7(63.6)$ & 0.8506 \\
\hline Extrafocal progression, $\mathrm{n}(\%)$ & $12(46.2)$ & $5(33.3)$ & $7(63.6)$ & 0.1257 \\
\hline \multicolumn{5}{|l|}{6 months after initial TACE } \\
\hline Number of patients in follow-up & 26 & 15 & 11 & \\
\hline Local recurrence, n (\%) & $18(69.2)$ & $10(66.7)$ & $8(72.7)$ & 0.7408 \\
\hline Extrafocal progression, n (\%) & $14(53.8)$ & $7(46.7)$ & $7(63.6)$ & 0.3912 \\
\hline
\end{tabular}

Abbreviations: CTACE, conventional transarterial chemoembolization; DEB-TACE, transarterial chemoembolization with drug-eluting beads; TACE, transarterial chemoembolization.

Survival rates comparing cTACE and DEB-TACE have been already investigated by prospective randomized controlled trials, in retrospective studies as well as in metaanalyses, and the results are heterogeneous (Table 5). Two of these studies showed a prolonged overall and median survival in patients treated with DEB-TACE, ${ }^{12,19}$ while the other studies could not demonstrate relevant differences. ${ }^{11,13,20,21}$ One study even showed a higher response rate and a longer time to progression after conventional chemoembolization. ${ }^{22}$ Our data did not show any differences in the overall and median survival, but there were differences in the median survival showing better results after cTACE in patients with multinodular or bilobar tumor burden and in patients having liver cirrhosis in Child-Pugh class A.

Investigating local recurrence of disease after 3 months and 6 months also did not reveal any differences between the study groups. Surprisingly, there was a trend toward more cases of extrafocal recurrence in the DEB-TACE group, although this difference was not statistically significant. This could be explained by the higher amount of multinodular HCCs in the DEB-TACE group.

As mentioned earlier, the safety of DEB could be confirmed in former studies. It was also shown that fewer side effects occurred after DEB-TACE in comparison to the conventional procedure. ${ }^{7,15}$ In contrast to these data, there was no trend for less toxic effects of the DEB in comparison to
cTACE in our analysis. This is in accordance with the data of adverse events published by the Precision Italia Study Group comparing DEB-TACE and cTACE in 177 patients, ${ }^{11}$ showing no differences in the rate of postinterventional fever, increased alanine transaminase, nausea, or liver function worsening. However, this randomized study revealed advantage of DEB-TACE in postprocedural abdominal pain. In contrast to this result, our analysis showed that DEB-TACEtreated patients experienced increased postinterventional pain and consecutively required statistically significant more pain medication within the first 3 days after the procedure. Although our data are limited by the small study population and its retrospective character, this issue was never described in previous studies comparing the two modalities of TACE. To avoid, that the extend of liver tissue treated with TACE of both modalities influenced these results, we performed a subgroup analysis of patients receiving TACE of an entire liver lobe or the whole liver. This subgroup analysis confirmed, that patients receiving DEB-TACE required significantly more opiate containing pain medication $(50.0 \%$ vs $8.3 \%$, $P=0.0289$ ). Postprocedural fever was not a factor for the administration of postoperative pain medication or nonsteroidal anti-inflammatory drug since there was no difference in the appearance of postinterventional fever in the two groups. The significant difference in pain medication with a fivefold higher application rate of opiates in the DEB-TACE group is

Table 5 Literature on cTACE versus DEB-TACE

\begin{tabular}{|c|c|c|c|c|c|c|c|c|}
\hline \multirow[t]{2}{*}{ Reference } & \multirow[t]{2}{*}{$\begin{array}{l}\text { Patients } \\
\text { (n) }\end{array}$} & \multicolumn{2}{|c|}{ Median survival (mo) } & \multicolumn{2}{|c|}{ Tumor response (\%) } & \multicolumn{2}{|c|}{$\begin{array}{l}\text { Time to progression } \\
\text { (mo) }\end{array}$} & \multirow[t]{2}{*}{ Adverse events } \\
\hline & & cTACE & DEB-TACE & cTACE & $\overline{\text { DEB-TACE }}$ & cTACE & $\overline{\text { DEB-TACE }}$ & \\
\hline Facciorusso et $\mathrm{a}^{22}$ & 249 & 39.0 & 32.0 & 85.3 & 74.8 & 17.0 & 11.0 & No differences \\
\hline Golfieri et al" & 177 & 28.0 & 29.0 & 74.1 & 74.7 & 9.0 & 9.0 & Less pain after DEB-TACE \\
\hline Kloeckner et $\mathrm{a}^{21}$ & 250 & 13.6 & 12.3 & $\mathrm{n} / \mathrm{a}$ & $\mathrm{n} / \mathrm{a}$ & $\mathrm{n} / \mathrm{a}$ & $\mathrm{n} / \mathrm{a}$ & No differences \\
\hline Song et $\mathrm{al}^{13}$ & 129 & 24.7 & 32.2 & 49.4 & 81.6 & 7.6 & 11.7 & No differences \\
\hline Dhanasekaran et al' ${ }^{\prime 2}$ & 71 & 3.8 & 13.4 & $\mathrm{n} / \mathrm{a}$ & $\mathrm{n} / \mathrm{a}$ & $\mathrm{n} / \mathrm{a}$ & $\mathrm{n} / \mathrm{a}$ & No difference in mortality \\
\hline
\end{tabular}

Abbreviations: cTACE, conventional transarterial chemoembolization; DEB-TACE, transarterial chemoembolization with drug-eluting beads; mo, months; n/a, not applicable. 
not explainable by the higher amount of multinodular tumor burden in the DEB-TACE group.

The major adverse events appeared in $9.4 \%$ of patients with no advantage for cTACE or DEB-TACE. In comparison to other study collectives, this rate seems quite high. However, this high 30-day mortality could be due to a high rate of not superselective procedures $(71.9 \%)$ in patients with a high tumor burden with consecutive higher risk of maldistribution of embolic agent or severe liver toxicity with consecutive liver failure.

\section{Conclusion}

Our study could not confirm superiority regarding tumor response and overall survival of DEB-TACE over cTACE. Surprisingly, a higher rate of postinterventional pain was detected and described for the first time after DEB-TACE.

\section{Acknowledgment}

This publication was supported by the Open Access Publication Fund of the University of Wuerzburg.

\section{Disclosure}

The authors report no conflicts of interest in this work.

\section{References}

1. Tiong L, Maddern GJ. Systematic review and meta-analysis of survival and disease recurrence after radiofrequency ablation for hepatocellular carcinoma. Br J Surg. 2011;98(9):1210-1224.

2. Cabrera R, Nelson DR. Review article: the management of hepatocellular carcinoma. Aliment Pharmacol Ther. 2010;31(4):461-476.

3. Bruix J, Sherman M, American association for the study of liver D. Management of hepatocellular carcinoma: an update. Hepatology. 2011;53(3):1020-1022.

4. Lo CM, Ngan H, Tso WK, et al. Randomized controlled trial of transarterial lipiodol chemoembolization for unresectable hepatocellular carcinoma. Hepatology 2002;35(5):1164-1171.

5. Llovet JM, Real MI, Montana X, et al. Arterial embolisation or chemoembolisation versus symptomatic treatment in patients with unresectable hepatocellular carcinoma: a randomised controlled trial. Lancet. 2002;359(9319):1734-1739.

6. Llovet JM, Bruix J. Systematic review of randomized trials for unresectable hepatocellular carcinoma: chemoembolization improves survival. Hepatology. 2003;37(2):429-442.

7. Lammer J, Malagari K, Vogl T, et al; PRECISION V Investigators. Prospective randomized study of doxorubicin-eluting-bead embolization in the treatment of hepatocellular carcinoma: results of the PRECISION V study. Cardiovasc Intervent Radiol. 2010;33(1):41-52.

Hepatic Medicine: Evidence and Research

\section{Publish your work in this journal}

Hepatic Medicine: Evidence and Research is an international, peerreviewed, open access journal covering all aspects of adult and pediatric hepatology in the clinic and laboratory including the following topics: Pathology, pathophysiology of hepatic disease; Investigation and treatment of hepatic disease; Pharmacology of drugs used for
8. Lewis AL, Taylor RR, Hall B, Gonzalez MV, Willis SL, Stratford PW. Pharmacokinetic and safety study of doxorubicin-eluting beads in a porcine model of hepatic arterial embolization. J Vasc Interv Radiol. 2006;17(8):1335-1343.

9. Lewis AL, Gonzalez MV, Leppard SW, et al. Doxorubicin eluting beads-1: effects of drug loading on bead characteristics and drug distribution. J Mater Sci Mater Med. 2007;18(9):1691-1699.

10. Hong K, Khwaja A, Liapi E, Torbenson MS, Georgiades CS, Geschwind JF. New intra-arterial drug delivery system for the treatment of liver cancer: preclinical assessment in a rabbit model of liver cancer. Clin Cancer Res. 2006;12(8):2563-2567.

11. Golfieri R, Giampalma E, Renzulli M, et al; PRECISION ITALIA STUDY GROUP. Randomised controlled trial of doxorubicin-eluting beads vs conventional chemoembolisation for hepatocellular carcinoma. Br J Cancer. 2014;111(2):255-264.

12. Dhanasekaran R, Kooby DA, Staley CA, Kauh JS, Khanna V, Kim HS. Comparison of conventional transarterial chemoembolization (TACE) and chemoembolization with doxorubicin drug eluting beads (DEB) for unresectable hepatocelluar carcinoma (HCC). J Surg Oncol. 2010;101(6):476-480.

13. Song MJ, Chun HJ, Song do S, et al. Comparative study between doxorubicin-eluting beads and conventional transarterial chemoembolization for treatment of hepatocellular carcinoma. $J$ Hepatol. 2012;57(6):1244-1250.

14. Hanish SI, Knechtle SJ. Liver transplantation for the treatment of hepatocellular carcinoma. Oncology (Williston Park). 2011;25(8):752-757.

15. Vogl TJ, Lammer J, Lencioni R, et al. Liver, gastrointestinal, and cardiac toxicity in intermediate hepatocellular carcinoma treated with PRECISION TACE with drug-eluting beads: results from the PRECISION V randomized trial. AJR Am J Roentgenol. 2011;197(4):W562-W570.

16. Varela M, Real MI, Burrel M, et al. Chemoembolization of hepatocellular carcinoma with drug eluting beads: efficacy and doxorubicin pharmacokinetics. J Hepatol. 2007;46(3):474-481.

17. Malagari K, Alexopoulou E, Chatzimichail K, et al. Transcatheter chemoembolization in the treatment of $\mathrm{HCC}$ in patients not eligible for curative treatments: midterm results of doxorubicin-loaded DC bead. Abdom Imaging. 2008;33(5):512-519.

18. Song MJ, Park CH, Kim JD, et al. Drug-eluting bead loaded with doxorubicin versus conventional Lipiodol-based transarterial chemoembolization in the treatment of hepatocellular carcinoma: a case-control study of Asian patients. Eur J Gastroenterol Hepatol. 2011;23(6):521-527.

19. Huang K, Zhou Q, Wang R, Cheng D, Ma Y. Doxorubicin-eluting beads versus conventional transarterial chemoembolization for the treatment of hepatocellular carcinoma. $J$ Gastroenterol Hepatol. 2014;29(5):920-925.

20. Gao S, Yang Z, Zheng Z, et al. Doxorubicin-eluting bead versus conventional TACE for unresectable hepatocellular carcinoma: a meta-analysis. Hepatogastroenterology. 2013;60(124):813-820.

21. Kloeckner R, Weinmann A, Prinz F, et al. Conventional transarterial chemoembolization versus drug-eluting bead transarterial chemoembolization for the treatment of hepatocellular carcinoma. BMC Cancer. 2015; $15: 465$.

22. Facciorusso A, Mariani L, Sposito C, et al. Drug-eluting beads versus conventional chemoembolization for the treatment of unresectable hepatocellular carcinoma. J Gastroenterol Hepatol. 2016;31(3):645-653.

the treatment of hepatic disease. Issues of patient safety and quality of care will also be considered. The manuscript management system is completely online and includes a very quick and fair peer-review system, which is all easy to use. Visit http://www.dovepress.com/ testimonials.php to read real quotes from published authors. 